\title{
Two cases of rare thyroid malignancy-case report
}

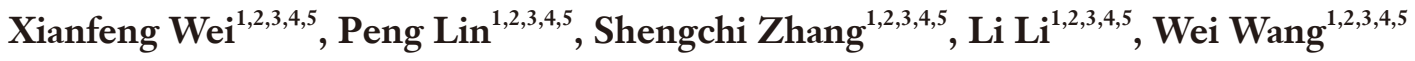 \\ ${ }^{1}$ Department of Otolaryngology Head and Neck, Tianjin First Central Hospital, Tianjin, China; ${ }^{2}$ Tianjin Institute of Otolaryngology, Tianjin, \\ China; ${ }^{3}$ Key Laboratory of Auditory Speech and Balance Medicine, Tianjin, China; ${ }^{4}$ Key Clinical Discipline of Tianjin Otolaryngology, Tianjin, \\ China; ${ }^{5}$ Otolaryngology Clinical Quality Control Centre, Tianjin, China \\ Correspondence to: Li Li, MB; Wei Wang, MD. Department of Otolaryngology-Head and Neck Surgery, Tianjin First Central Hospital, 24 Fukang \\ Road, Nankai district, Tianjin 300192, China; Tianjin Institute of Otolaryngology, Tianjin 300192, China; Key Laboratory of Auditory Speech \\ and Balance Medicine, Tianjin 300192, China; Key Clinical Discipline of Tianjin Otolaryngology, Tianjin 300192, China; Otolaryngology Clinical \\ Quality Control Centre, Tianjin 300192, China. Email: liyueban1@163.com; doctorwangw@sina.com.
}

\begin{abstract}
We report 2 cases of rare thyroid malignancy: angiosarcoma and myoepithelial carcinoma (MC). Thyroid angiosarcomas (TAS) is extremely rare and comprises less than $1 \%$ of primary thyroid cancer worldwide. MC usually presents as a slow-growing painless mass arising in the salivary glands. It has not been reported in the thyroid gland. The first case describes a 59-year-old patient who was admitted to hospital with the discovery of thyroid nodule for 1 month. The tumor thrombus was found in the left internal jugular vein and superior thyroid artery during the operation. Diagnosis of angiosarcoma of the thyroid was based on positive endothelial markers such as thrombomodulin and CD31 after total thyroidectomy. The left internal jugular vein, left recurrent laryngeal nerve and anterior cervical banding muscle were invaded by thyroid tumor. No lymph node metastasis was observed. The patient died after 4 years. The second case describes a 55-year-old woman who presented with the discovery of thyroid nodule for 1 month. Right thyroid lobectomy and right neck lymph node functional dissection were carried out. The results from postoperative pathology revealed that papillary carcinoma in right lobe of thyroid and MC next to thyroid were found. Besides, the metastasis of MC was observed at right II-IV level and right VI level. Five years later, the patient was re-admitted to hospital, primarily due to the discovery of anterior cervical tumor for one year. Then, she underwent left thyroid lobectomy and right tumor resection. Postoperative routine pathology showed recurrent $\mathrm{MC}$ in the right thyroid. After surgery and radiotherapy, the patient was followed up for 2 years. Angiosarcoma and myoepithelioma should be kept in mind in diagnosis of thyroid malignant tumor.
\end{abstract}

Keywords: Thyroid gland; angiosarcoma; myoepithelial carcinoma (MC); thyroidectomy; case report

Submitted Jul 12, 2020. Accepted for publication Nov 04, 2020.

doi: $10.21037 /$ gs-20-601

View this article at: http://dx.doi.org/10.21037/gs-20-601

\section{Introduction}

Mesenchymal origin of primary thyroid angiosarcoma (TAS) is extremely rare and comprises less than $1 \%$ of primary thyroid cancer worldwide (1). The diagnosis of TAS is usually determined by cytology after thyroidectomy, which is challenging because of the lack of cells, the appearance of necrotic tissue, unawareness of the disease due to rarity. Salivary gland myoepithelial carcinoma (MC) or malignant myoepithelioma is a rare entity. MC usually presents as a slow-growing painless mass arising in the parotid gland, but may involve other salivary glands. It has not yet been reported in the thyroid gland. This tumour may be particularly locally aggressive, but its clinical and biological features are not yet fully understood. Other published data (2) suggest it is likely to be a high-grade neoplasm, consistent with its aggressive behaviour.

We present the following cases in accordance with the CARE reporting checklist (available at http://dx.doi. org/10.21037/gs-20-601). 

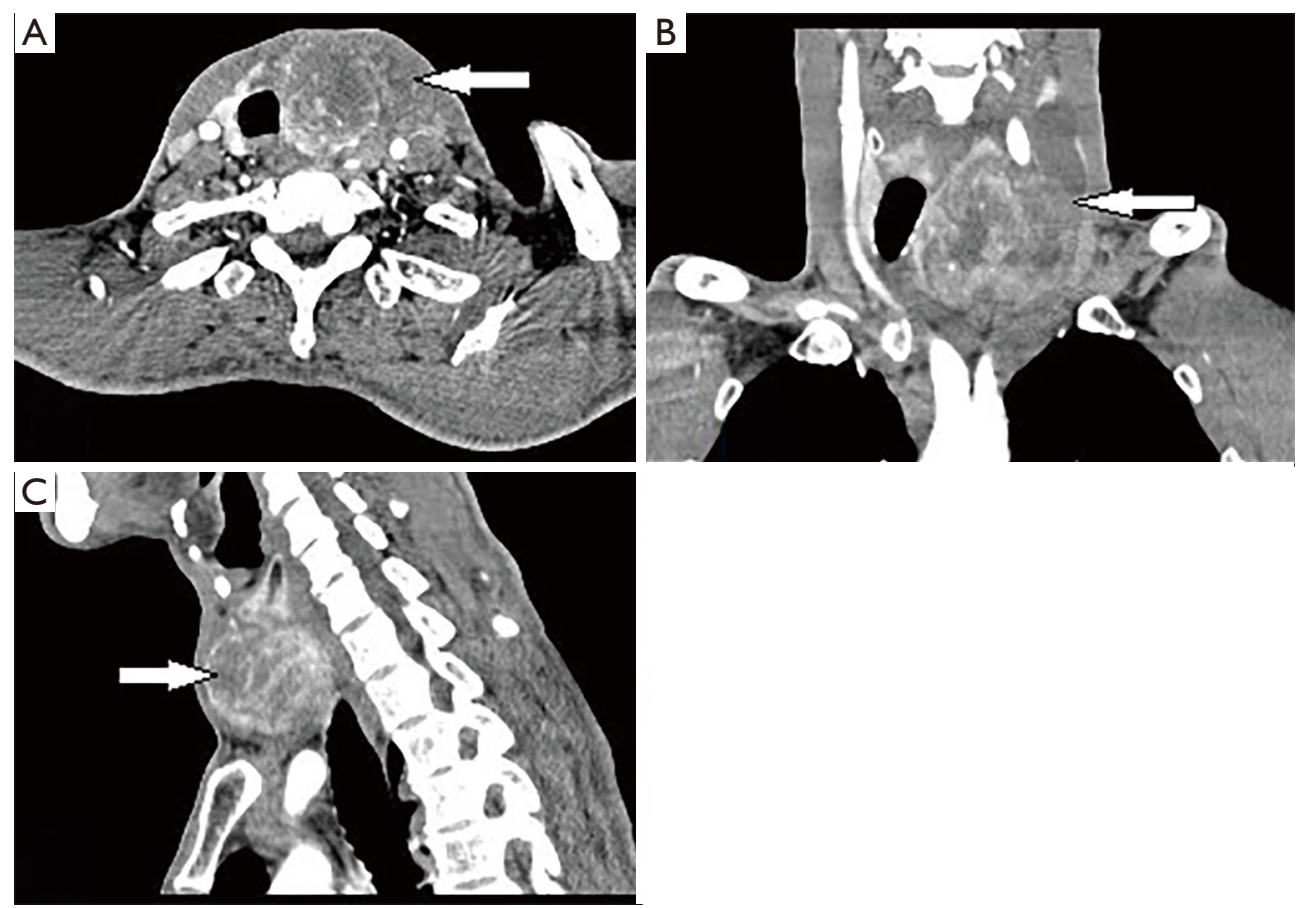

Figure 1 The enhanced computed tomography (CT) in Case 1 shows a large non-homogenous mass with ill-defined margins in left lobe of the thyroid and the vein tumor thrombus formation in left jugular. (A) Horizontal scan. (B) Coronal scan. (C) Sagittal scan.

\section{Case presentation}

All procedures performed in studies involving human participants were in accordance with the ethical standards of Tianjin First Central Hospital and with the Helsinki Declaration (as revised in 2013). Written informed consent was obtained from the patients.

\section{Case 1}

The patient (male, 59 years old) was hospitalized on July 2013, mainly due to the discovery of progressively enlarged mass at his anterior neck accompanied by difficulty in swallowing for one month. He had a history of diabetes mellitus and no other significant abnormalities were detected. Physical examination showed a moderately hard mass in the left lobe of thyroid, about $20 \mathrm{~cm} \times 20 \mathrm{~cm}$, with poor activity and unclear boundary. The ultrasonography of thyroid revealed a predominantly cystic or solid nodule with calcification in the left lobe and multiple solid nodules in the right lobe, possible thrombus formation in the left internal jugular vein and its branches. Enlarged lymph nodes were found at the left neck by ultrasound. The result of thyroid function test was within normal limits. As shown in Figure 1, the space-occupying lesion in left thyroid was detected by the enhanced computed tomography (CT), suggesting the possibility of thyroid cancer and the vein tumor thrombus formation in left jugular. The fine needle aspiration was not performed because the great possibility of thyroid malignancy judged by imaging. Subsequently, we explained it to the patient and his family and suggested that PET-CT should be performed to examine whether there was distant metastasis. After consultation, the patient decided to operate first and refused PET examination. Total thyroidectomy, intraoperative frozen pathology and double neck lymph node dissection were performed under general anesthesia. The results showed that the size of left lobe of thyroid gland was $20 \mathrm{~cm} \times 20 \mathrm{~cm} \times 10 \mathrm{~cm}$. Moreover, the tumor infiltrated the thyroid capsule and closely adhered to peripheral tissues. Meanwhile, the left internal jugular vein, left recurrent laryngeal nerve and anterior cervical banding muscle were invaded by thyroid tumor. Consequently, the common carotid artery was squeezed outward and the tumor thrombus was detected in the left internal jugular vein and superior thyroid artery. The size of right lobe of thyroid was $6 \mathrm{~cm} \times 5 \mathrm{~cm} \times 3 \mathrm{~cm}$ and contained multiple sclerotic nodules. A series of enlarged lymph nodes were observed in the level 2, 3 and 4 of the left carotid sheath. 

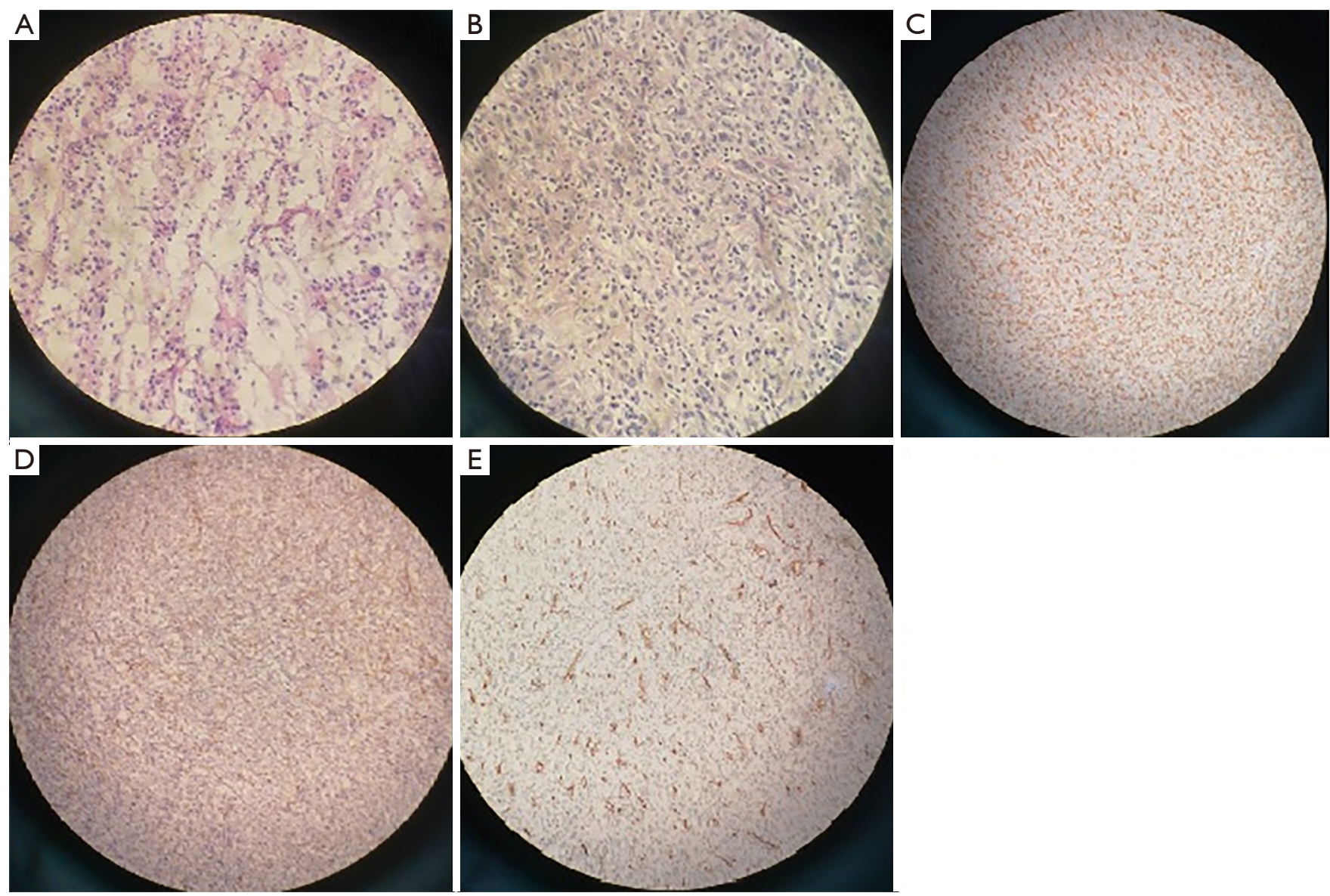

Figure 2 Case 1 pathological image. (A) Frozen pathology in operation shows malignant tumors (400×; H\&E stain). A small dispersive group of large, atypical, epithelioid cells with well-defined cell borders, pleomorphic and vesicular, central nuclei and eosinophilic cytoplasm. (B) Postoperative pathology proves to be hemangiosarcoma (400x; H\&E stain). Tumors featured a solid pattern of growth with cells arranged in sheets and nests with no intervening stroma. The neoplastic cells were epithelioid, spindled or polygonal-shaped with abundant eosinophilic cytoplasm and round, large, vesicular nuclei containing prominent nucleoli. (C) Postoperative immunohistochemical slide (200x): VIII(+). (D) Immunohistochemical slide (200x): CD31(+). (E) Immunohistochemical slide (200x): CD34(+).

Furthermore, the enlarged lymph nodes in level 6 of the left carotid sheath were fused with the tumor. The malignancy in the left lobe was found by intraoperative frozen pathology analysis (Figure 2A). However, the characteristic had not been identified. Postoperative pathology indicated the presence of hemangiosarcoma in the left lobe of the thyroid gland (Figure $2 B$ ) and it was confirmed by immunohistochemical markers (Figure 2C,D,E). In addition, no lymph node metastasis was observed. Therefore, the postoperative radiotherapy was recommended, but it was refused by the patient. The patient was followed up for 4 years and died of multiple metastases from the hands, ribs, axillary lymph nodes and lung in May 2017.

\section{Case 2}

The patient (female, 55 years old) was admitted to hospital on May 2013, mainly because of the presence of anterior cervical tumor for more than one month. There was a hard mass about $4 \mathrm{~cm} \times 5 \mathrm{~cm}$ in front of the right neck of the patient, moving up and down with swallowing, with the unclear boundary. There was no dyspnea, dysphagia and hoarseness. Thyroid ultrasonography showed that there was a hypoechoic mass (about $53 \mathrm{~mm} \times 30 \mathrm{~mm} \times 29 \mathrm{~mm}$ ) in the right lobe of the thyroid (Figure $3 A, B$ ). However, the boundary was unclear. The CT suggested that the tumor observed in right lobe of the thyroid involved the whole thyroid (Figure 3C). The thyroid function was normal. A 

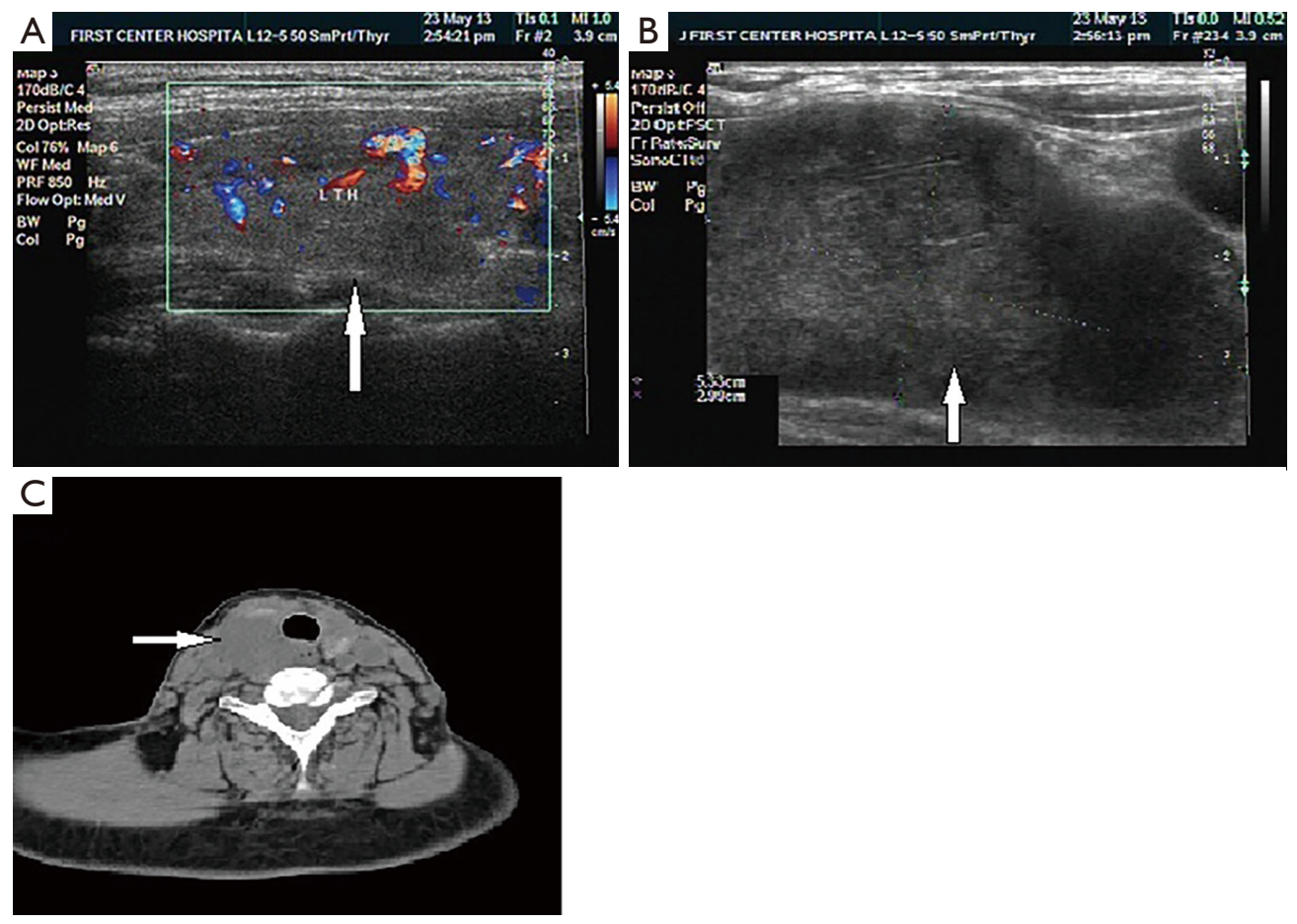

Figure 3 Imaging shows right lobe lesions in the thyroid gland in Case 2. (A) The images showing a predominantly hypoechoic mass with partial indistinct margin within the right thyroid lobe. (B) Local amplification showing right lobe lesions of the thyroid gland on ultrasound. (C) Nonenhanced CT imaging shows an irregular lobulated lesion with ill-defined margins in thyroid right lobe and the unclear demarcation to esophagus.

fine needle aspiration cytology (FNAC) of the mass was performed which yielded epithelial cells and myoepithelial cells which was lobulated, tubular and solid area mixed, rendering a diagnosis difficult. Right thyroid lobectomy, intraoperative frozen pathology, and right neck lymph node functional dissection were carried out under general anesthesia. The results showed that the size of right lobe of thyroid gland was $4 \mathrm{~cm} \times 3 \mathrm{~cm} \times 2 \mathrm{~cm}$ and approximatively $1 \mathrm{~cm}$ tumor could be palpable. On the lateral side of the thyroid gland, a tumor was lobulated, hard in quality and closely adhered to the thyroid gland and esophageal wall and it was cut out completely. Furthermore, the tumor was observed to be about $7 \mathrm{~cm} \times 5 \mathrm{~cm} \times 3 \mathrm{~cm}$ in size and the tumor did not occur in the left lobe of thyroid. Intraoperative frozen pathology showed the origin of neck tumor was unclear and it was the papillary carcinoma in the right lobe of thyroid. The results from postoperative pathology revealed that papillary carcinoma in right lobe of thyroid and MC next to thyroid were found (Figure 4). Besides, the metastatic tumor of myoepithelioma was observed at right II level (1/6), right III level (3/7), right IV level (2/9) and right VI level (2/9). Ultrasound of parotid gland showed no abnormality after operation.

Five years later, the patient was re-admitted to hospital, primarily due to the discovery of anterior cervical tumor for one year. The patient had received the traditional Chinese herbal medicine therapy since her last discharge and no abnormality was found within 1 year. After that, the patient did not receive re-examination. However, a tumor about $2 \mathrm{~cm} \times 3 \mathrm{~cm}$ in right neck was detected one year ago, which was hard and had the clear boundary. Thyroid $\mathrm{B}$ ultrasound was conducted and found that there was a low echo mass in the right neck after thyroidectomy. The characteristics of this mass were as follows: irregular shape, clear boundary, inhomogeneous, gravel-like strong echo spots, rich blood supply, hardness lower than the left lobe gland and $4 \mathrm{~B}$ grade of Thyroid imaging reporting and data system (TI-RADS) classification. Meanwhile, there were multiple nodules in the left lobe of thyroid $(>3$, TIRADS grade 3). The thyroid function was in normal range and the patient used to be in good health. As shown in Figure 5, the magnetic resonance suggested that a tumor 

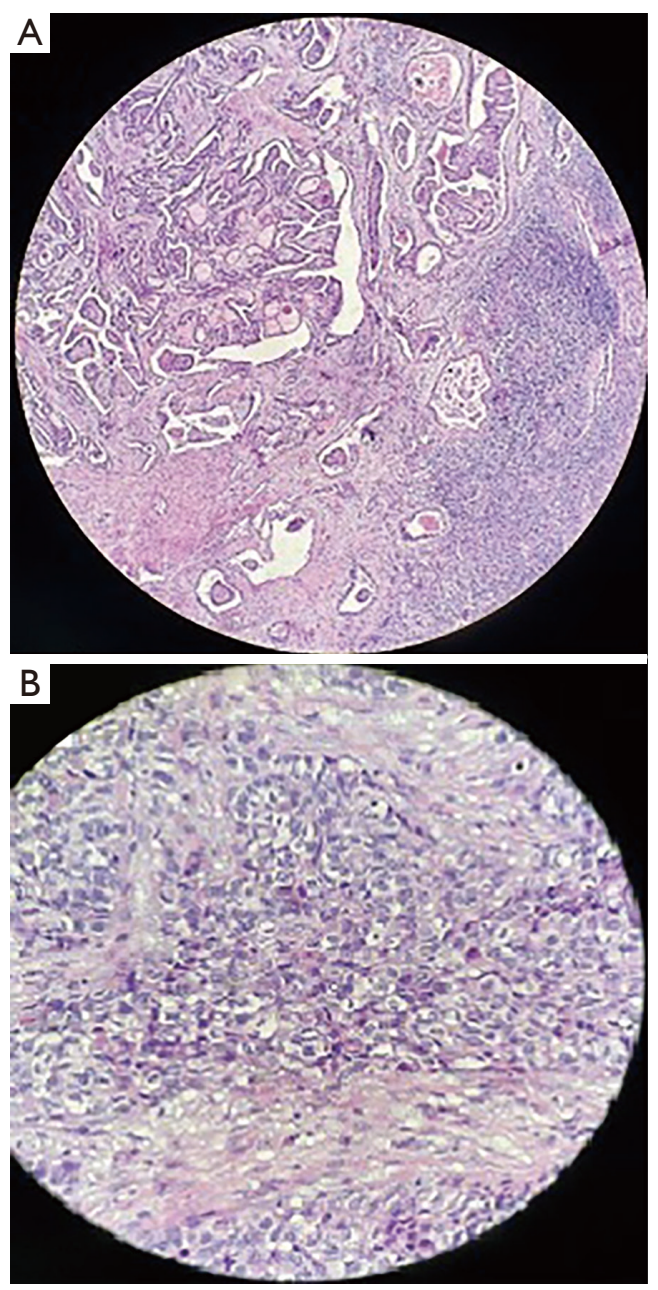

Figure 4 The first operation pathology in case 2. (A) Papillary carcinoma in right lobe of thyroid (100x; H\&E stain). It was diagnosed by nuclear overcrowding, ground glass nuclei, arborizing papillary processes, nuclear grooves. (B) Myoepithelial carcinoma next to the thyroid gland (400x; H\&E stain). It was a mixture of spindle-shaped, clear, and epithelioid cells.

on the right side of the thyroid with a clear boundary and uneven signal intensity. Then, left thyroid lobectomy, right tumor resection and intraoperative frozen pathology were performed under general anesthesia. The results of frozen pathology showed that there was a malignant tumor in right thyroid (Figure 6A). Therefore, the medullary carcinoma was considered. Postoperative routine pathology showed recurrent $M C(3 \mathrm{~cm} \times 4 \mathrm{~cm} \times 5 \mathrm{~cm})$ in the right thyroid (Figure 6B), the postoperative immunohistochemistry typical for myoepithelial tumor cells in the form of Figure $6 C, D, E, F$, papillary carcinoma (diameter $0.1 \mathrm{~cm}$ ) in the left lobe of thyroid with Hashimoto's disease were found and no metastatic carcinoma in the anterior median cervical lymph node was observed. Finally, radiotherapy with a 6-MV medical linear accelerator (60 Gy/30 fractions) with $1 \mathrm{~cm}$ margin around the primary tumour was undertaken and no tumor recurrence was found during the follow-up for two years.

\section{Discussion}

Angiosarcoma is a rare malignant tumor belonging to vascular endothelium and characterized by the irregularly anastomosed angiogenic sarcoma (3). Its etiology is yet unknown. Approximately a quarter of the location of this disease was in the head and neck. Additionally, the common pathogenesis sites also included the skin, soft tissue, retroperitoneal space, limbs, breast and internal organs (3). Metastasis frequently occurred at lung, bone, kidney, adrenal gland and regional lymph nodes (3). TAS was characterized by its prevalence in Alpine regions of Central Europe. In most cases, TAS occurred in patients over 40 years of age, with a peak incidence for the group aged 60-79 years, with a female predominance. In most patients, TAS manifested clinically as a painless goiter. It can be accompanied by cough, dyspnea and dysphagia because of compression or infiltration to the trachea or esophagus. The size of tumor was variable, ranging from 2.5 to $14.7 \mathrm{~cm}$ in its greatest dimension, and most tumors were $\geq 5 \mathrm{~cm}$ (4). Most patients had no local or distant metastases at the time of diagnosis. The identified radiological features (ultrasound, CT and magnetic resonance imaging) are non-specific and tumor can be misdiagnosed as other malignant thyroid tumors, such as primary carcinoma or lymphoma (5). Histologically, the morphology of TAS may range from well-differentiated forms, to poorly differentiated tumors with solid growth of spindle and/or epithelioid cells (6).

Different malignant tumor can occur in thyroid. Medullary thyroid carcinoma originates from parafollicular cells of thyroid. Elevated serum calcitonin and carcinoembryonic antigen are specific serum markers of medullary thyroid carcinoma. It is often associated with RET gene mutation. Anaplastic carcinoma is a high degree of malignancy, with unclear boundary and rapid progress. It often invades important tissues or organs such as larynx, trachea, esophagus and so on. The primary foci of metastatic carcinoma of thyroid mainly originated from lung cancer (43\%), followed by breast cancer (9\%) 

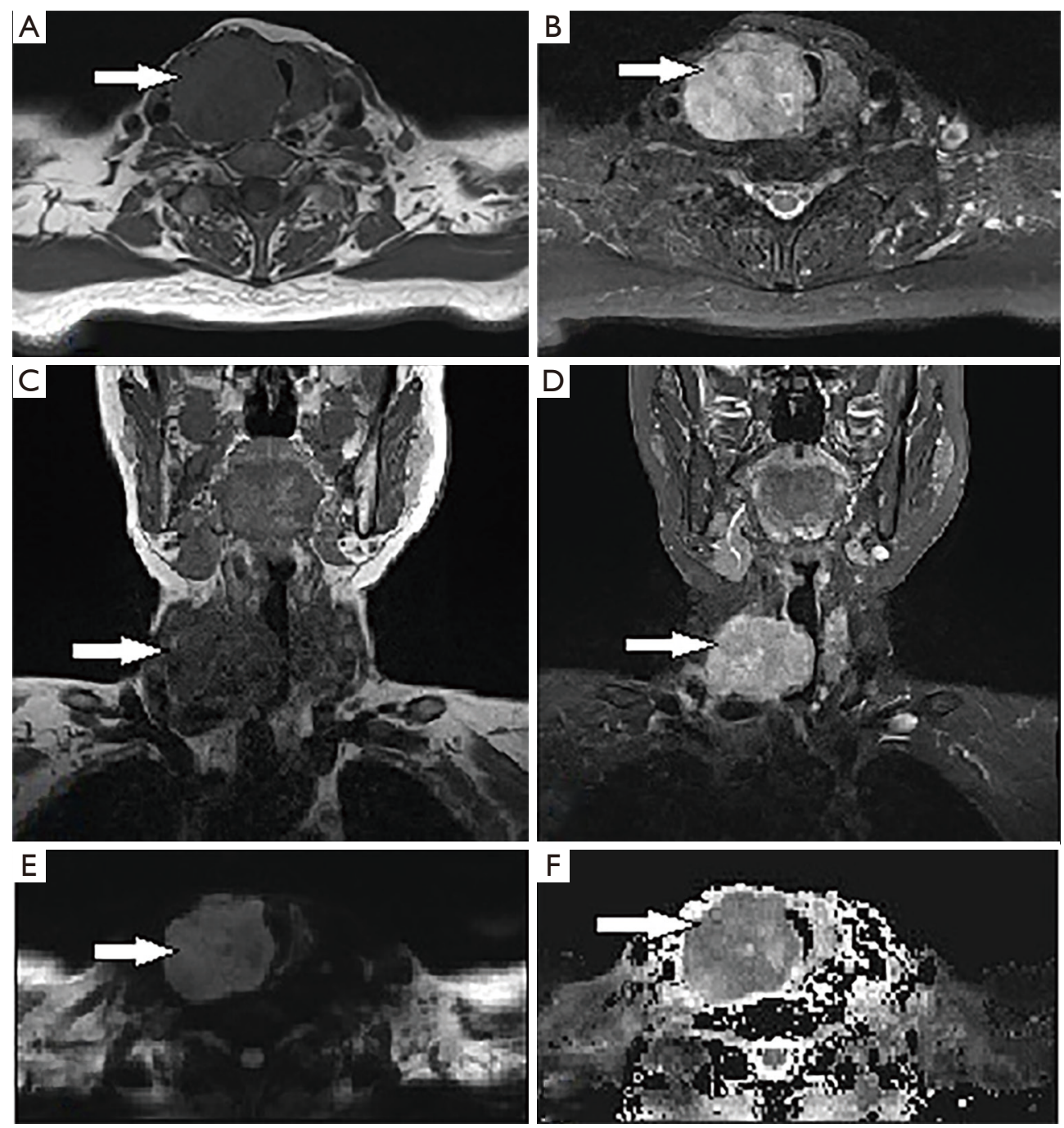

Figure 5 Magnetic resonance scan in Case 2 shows clumpy mass with equal T1, long T2, high diffusion weighted imaging (DWI) and low apparent diffusion coefficient (ADC) signals in the area after excision of the right gland lobe, which was irregular edges and unclear border. (A) T1 weighted image (T1WI) in horizontal scan. (B) T2 weighted image (T2WI) in horizontal scan. (C) T1WI in coronal scan. (D) T2WI in coronal scan. (E) DWI. (F) ADC.

and gastric cancer (8\%) (7), often accompanied by clinical manifestations of the primary lesions. The positive immunostaining of thyroglobulin and thyroid transcription factor-1 indicated the primary thyroid cancer, which often did not express in metastatic thyroid carcinoma, only expressing specific markers related to the tissue of origin. The diagnosis of angiosarcoma is now made easy by the availability of several antigenic markers. The existing evidence suggests that thrombomodulin and CD31 acts as the most reliable and powerful diagnostic marker (1), the tumor cells are positive for ERG, Fli1, CD34, factor VIII and CKpan to different extents (2). The neoplastic cells showed positive immunoreaction for factor VIII, CD31, CD34, respectively, in Figure 2C,D,E. The surgery and radiotherapy have been advocated as the main treatment in the recent years (8), the role of chemotherapy remains to be elucidated (6). The overall prognosis is dismal, and most patients die from disease in less than 6 months (9). As it generally has poor prognosis, multiple lesions, positive surgical margin, external diameter size of tumor $>5 \mathrm{~cm}$, mitosis rate $>3 \mathrm{HPF}$, depth of invasion $>3 \mathrm{~mm}$, tumor recurrence and metastases (8). 

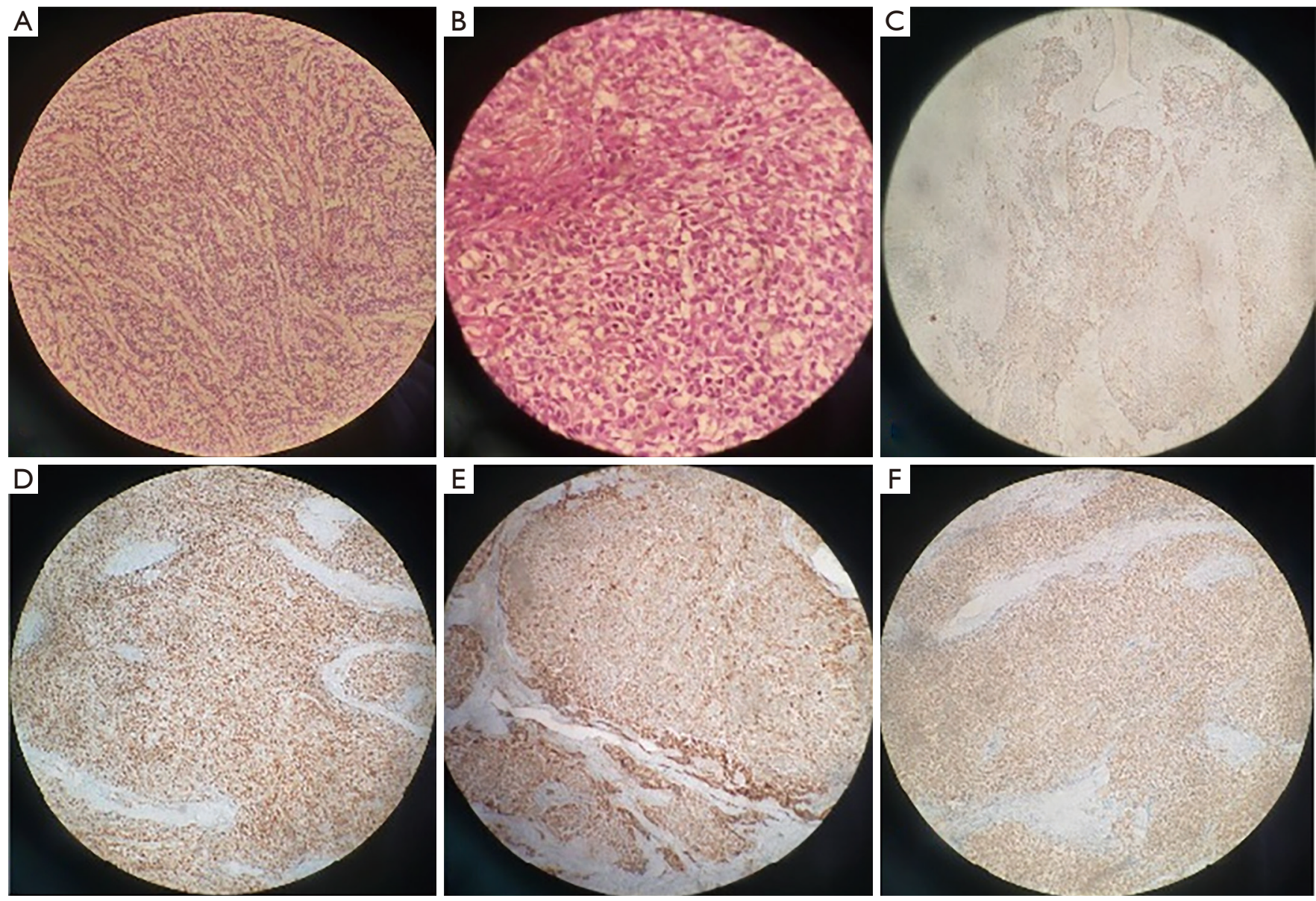

Figure 6 The second operation pathology in case 2. (A) Frozen pathology in operation shows malignant tumors (100×; H\&E stain). The circular or the spindle-shaped cells were divided by fibrous septa, arranged in small leafy or trabecular shapes, with round or oval cell nucleus and granulated cytoplasm. (B) Postoperative conventional pathology proves to be recurrent myoepithelial carcinoma (400x; H\&E stain). An inner layer of duct lining cells and an outer layer of clear cells, which typically form double-layered duct-like structures. (C) Postoperative immunohistochemical slide (100x): CK(+). (D) Immunohistochemical slide (100x): CyclinD1(+). (E) Immunohistochemical slide (100x): Galectin3(+). (F) Immunohistochemical slide (100x): S100(+).

Malignant MCs is a rare mass tumor that makes up 1 to 2 percent of all salivary gland neoplasms (10). It has not been reported in the thyroid gland. There is a female predominance, with a peak occurrence in the seventh decade (11). It usually presents as painless and slowgrowing mass in parotid gland, oral cavity or neck, and its clinical and biological behavior has not been fully elucidated. Myoepithelial tumors may contain four cell types thought to represent various stages in myoepithelial cell differentiation (12). These cell types include spindleshaped, epithelioid, plasmacytoid and clear cells, or combinations thereof. The current World Health Organization salivary gland classification (13) considered
MC to be an intermediate-to-high grade malignancy, which was invasive and easy to recurrence. There are few articles on the imaging characteristics of myoepithelial cancer. Nonenhanced CT imaging showed an irregular lobulated or multinodular lesion with ill-defined margins and inhomogeneous attenuation and punctate calcification in a minority of the lesions. The enhanced CT imaging results revealed moderate and intense inhomogeneous enhancement, including cystic and slit-like regions with no enhancement, intense nodular enhancement, and small tortuous vessels in the arterial phase (14). Preoperative CT and MRI examinations are very important, surgeon can make a more accurate assessment of the extent and nature 
of the tumor, help to develop a reasonable surgical plan (15). Cytomorphologically, the identification of MCs depends on the presence of infiltrative growth, mitotic count, cellular polymorphism, tumor necrosis or a combination thereof (16). Immunohistochemical studies play a key role in the confirmation of myoepithelial differentiation (17). Numerous studies (13) have demonstrated that S100, vimentin and CK are currently the most sensitive markers for tumor myoepithelium. In this work, the immunohistochemical staining assay suggested that the CK, CyclinD1, Galectin3, S100 and syn were all expressed in myoepithelial cells (Figure 6C,D,E,F). Radical surgery is still the preferred method for the treatment of the disease. Postoperative chemotherapy or radiotherapy may help to prevent metastasis and recurrence and has not yet reached a consensus (16). The rate of recurrence and metastasis accounts for approximately $60 \%$ (18). The patient was satisfied with the treatment.

\section{Conclusions}

We report 2 cases of rare thyroid malignancy: angiosarcoma and $\mathrm{MC}$, which can be used as baseline data for future reference and research for this cancer.

\section{Acknowledgments}

Funding: None.

\section{Footnote}

Reporting Checklist: The authors have completed the CARE reporting checklist. Available at http://dx.doi.org/10.21037/ gs-20-601

Peer Review File: Available at http://dx.doi.org/10.21037/gs20-601

Conflicts of Interest: All authors have completed the ICMJE uniform disclosure form (available at http://dx.doi. org/10.21037/gs-20-601). The authors have no conflicts of interest to declare.

Ethical Statement: The authors are accountable for all aspects of the work in ensuring that questions related to the accuracy or integrity of any part of the work are appropriately investigated and resolved. All procedures performed in studies involving human participants were in accordance with the ethical standards of Tianjin First Central Hospital and with the Helsinki Declaration (as revised in 2013). Written informed consent was obtained from the patients.

Open Access Statement: This is an Open Access article distributed in accordance with the Creative Commons Attribution-NonCommercial-NoDerivs 4.0 International License (CC BY-NC-ND 4.0), which permits the noncommercial replication and distribution of the article with the strict proviso that no changes or edits are made and the original work is properly cited (including links to both the formal publication through the relevant DOI and the license). See: https://creativecommons.org/licenses/by-nc-nd/4.0/.

\section{References}

1. Kondapalli A, Redd L, DeBlanche L, et al. Primary angiosarcoma of thyroid. BMJ Case Rep 2019;12:e228862.

2. Li H, Li HX, Zhu Y, et al. Clinicopathologic study of primary angiosarcoma of thyroid. Zhonghua Bing Li Xue Za Zhi 2016;45:364-7.

3. Shen Z, Mao W, Zhang W. Retrospective analysis for 75 cases of angiosarcoma. Chin J Diffic and Compl Cas 2007;6:469-71.

4. Huang SH, Wu SC. Primary Angiosarcoma of the Thyroid in an Asian Woman: A Case Report with Review of the Literature. Case Rep Pathol 2020;2020:9068506.

5. Surov A, Gottschling S, Wienke A, et al. Primary Thyroid Sarcoma: A Systematic Review. Anticancer Res 2015;35:5185-91.

6. Marina M, Corcione L, Serra MF, et al. Primary Epithelioid Angiosarcoma of the Thyroid in a Patient Occupationally Exposed to Radiations. Front Endocrinol (Lausanne) 2018;9:577.

7. Zhang L, Liu Y, Li X, et al. Metastases to the thyroid gland: A report of 32 cases in PUMCH. Medicine (Baltimore) 2017;96:e7927.

8. Kharkar V, Jadhav P, Thakkar V, et al. Primary cutaneous angiosarcoma of the nose. Indian J Dermatol Venereol Leprol 2012;78:496-7.

9. Wick MR, Eusebi V, Lamovec J, et al. Angiosarcoma. In: Lloyd RV, Osamura RY, Kloeppel G, et al. editors. WHO Classification of tumours of endocrine organs. Lyon, France: IARC Press, 2017:129-32.

10. Zeitouni NC, Huang DD, Row D. An Unusual Presentation and Aggressive Course of Metastatic Myoepithelial Carcinoma. J Clin Aesthet Dermatol 
2019;12:46-8.

11. Seifert G, Sobin LH. Epithelial-Myoepithelial Carcinoma. In World Health Organization International Histo-logical Classification of Tumours. Histological Typing of Salivary Gland Tumours. 2nd Edition, Springer-Veflag Berlin, 1991:23-4.

12. Seethala RR, Barnes EL, Hunt JL. EpithelialMyoepithelial Carcinoma: A Review of the Clinicopathologic Spectrum and Immunophenotypic Characteristics in 61 Tumors of the Salivary Glands and Upper Aerodigestive Tract. Am J Surg Pathol 2007;31:44-57.

13. Vilar-Gonzalez S, Bradley K, Rico-Perez J, et al. Salivary gland myoepithelial carcinoma. Clin Transl Oncol 2015;17:847-55.

14. Yue D, Feng W, Ning C, et al. Myoepithelial carcinoma of the salivary gland: pathologic and CT imaging characteristics (report of 10 cases and literature

Cite this article as: Wei X, Lin P, Zhang S, Li L, Wang W. Two cases of rare thyroid malignancy-case report. Gland Surg 2021;10(1):386-394. doi: 10.21037/gs-20-601 review). Oral Surg Oral Med Oral Pathol Oral Radiol 2017;123:e182-7.

15. Yamada H, Kawaguchi K, Yagi M, et al. Epithelialmyoepithelial carcinoma of the submandibular gland with a high uptake of $18 \mathrm{~F}-\mathrm{FDG}$ : a case report and image diagnosis. Oral Surg Oral Med Oral Pathol Oral Radiol Endod 2007;104:243-8.

16. Nagao T, Sugano I, Ishida Y, et al. Salivary gland malignant myoepithelioma: a clinicopathologic and immunohistochemical study of ten cases. Cancer 1998;83:1292-9.

17. Zhang L, Wang D, Ren Y, et al. Myoepithelial carcinoma of the parotid gland with bilateral thyroid involvement: A case report and review of the literature. Oncol Lett 2010;1:1079-82.

18. Savera AT, Sloman A, Huvos AG, et al. Myoepithelial carcinoma of the salivary glands: a clinicopathologic study of 25 patients. Am J Surg Pathol 2000;24:761-74. 